\title{
Tax Evasion and Multi-Agent-Based Model on Various Topologies
}

\author{
F. W. S. Lima \\ Dietrich Stauffer Computational Physics Lab \\ Departamento de Física, Universidade Federal do Piauí \\ Teresina-PI, 64049-550 Brazil \\ fwslima@gmail.com
}

Received 16 December 2016

Accepted 3 May 2017

Published 30 June 2017

\begin{abstract}
In this work, we use Monte-Carlo simulations to study the control of the fluctuations for tax evasion in the economics model proposed by [G. Zaklan, F. Westerhoff and D. Stauffer, J. Econ. Interact. Coordination. 4 (2009) 1; G. Zaklam, F.W.S. Lima and F. Westerhofd, Physica A 387 (2008) 5857.] via a nonequilibrium model with two states $(-1,+1)$ and a noise $q$ proposed for [M. J. Oliveira, J. Stat. Phys. 66 (1992) 273] and known as Majority-Vote model (MVM) and Sánchez-López-Rodríguez model on communities of agents or persons on some topologies as directed and undirected Barabási-Albert networks and Erdös-Rényi random graphs, Apollonian networks, directed small-world networks and Stauffer-Hohnisch-Pittnauer networks. The MVM is applied around the noise critical $q_{c}$ to evolve the Zaklan model.
\end{abstract}

Keywords: Networks; econophysics; majority vote; nonequilibrium.

\section{Introduction}

The tax evasion remains to be a major difficulty facing governments, ${ }^{1-4}$ and experimental evidence provided by Gächter ${ }^{5}$ indeed suggests that tax payers tend to condition their decision regarding whether to pay taxes or not on the tax evasion decision of the members of their neighborhood. Frey and Torgler ${ }^{6}$ also showed empirical evidence on the relevance of conditional cooperation for tax morale. Then realistic economics models of tax evasion have been and are being proposed to study the problem of the tax evasion. In this context, Zaklan et al. ${ }^{7,8}$ developed an economics model to study the problem of tax evasion dynamics using the Ising model through Monte-Carlo simulations with the Glauber and heatbath algorithms (that obey detailed-balance equilibrium) to study the proposed model. The Zaklan model

This is an Open Access article published by World Scientific Publishing Company. It is distributed under the terms of the Creative Commons Attribution 4.0 (CC-BY) License. Further distribution of this work is permitted, provided the original work is properly cited. 
has been robust because this can be studied using equilibrium dynamics of Ising model and also through the nonequilibrium Majority-Vote model (MVM) ${ }^{9}$ and on various topologies cited above giving the same behavior regardless of dynamics or topology used here.

Simulations on both undirected and directed scale-free networks, ${ }^{10-16}$ random graphs ${ }^{17,20}$ and social networks, ${ }^{21,22,25}$ have attracted the interest of researchers from various areas. These complex networks have been studied extensively by Lima et al. in the context of magnetism (MVM, Ising, and Potts model), ${ }^{26-29}$ econophysics ${ }^{8,30}$ and sociophysics. ${ }^{31}$ Lima ${ }^{32}$ made an analysis of tax evasion dynamics with the Zaklan model on square lattice (SL) using MVM for their temporal evolution under different enforcement regimes. They showed that the MVM model is also capable of controlling the different levels of the tax evasion as it was made by Zaklan et al. ${ }^{8}$ using Ising model on various structures: SL, Voronoi-Delaunay random lattice, BarabásiAlbert (BA) network and Erdös-Rényi (ER) graphs; we discuss the resulting tax evasion dynamics. This was the first application of the MVM model to a real system, in this case applied to the economy. Hokamp and Pickardt, ${ }^{33}$ and Pickhardt and Seibold $^{34}$ argued that any agent-based tax evasion model may according to their characterization fall into the economics domain or into the econophysics domain. Then, Pickhardt and Seibold classify the Zaklan model as a econophysics model because the model evolves by statistical mechanics using an Ising model or MVM. Many social and economic networks in the real world exhibit directed relations. This may be modeled by including directed links in the corresponding complex network model. In this context, Lima ${ }^{35}$ proposed to study the problem of the tax evasion on directed Small-World (DSW) network via a nonequilibrium dynamics model $^{11}$ with the objective to make the Zaklan model as realistic as possible. This is known as Sánchez-López-Rodríguez (SRL) model, ${ }^{11}$ where each agent has an opinion in the presence of a social noise $(q)$ as in the original MVM. ${ }^{9}$ This model is based on the knowledge that people do not live in a social equilibrium, and any rumor or gossip can lead to a government or market chaos.

In the present work, we make a brief review of the study of the tax evasion using the Zaklan model, ${ }^{7}$ since its creation until the present moment, on the various topologies cited above.

\section{Networks and Random Graphs}

\subsection{Directed and undirected Barabási-Albert network}

- Undirected Barabási-Albert (UBA) networks

The UBA network ${ }^{12,13}$ is grown such that the probability of a new node to be connected to one of the already existing nodes is proportional to the number of the previous connections to this already existing node: the rich get richer. In this way, each new node selects exactly $m$ old nodes as neighbors. If a new node selects randomly $m$ old nodes as neighbors, then the $m$ old nodes are added to a long array 
of node indices called the Kertész list, and the new node is also added $m$ times to that list. At the start of the network growth, this Kertész list is empty. The above random selections are made by selecting $m$ random nodes from the Kertész list. The neighbor relations were such that if $\mathrm{A}$ has $\mathrm{B}$ as a neighbor, $\mathrm{B}$ has $\mathrm{A}$ as a neighbor.

\section{- Directed Barabási-Albert (DBA) networks}

In DBA networks, the network itself is produced in the undirected BarabásiAlbert networks way. ${ }^{14,15}$ When interacting agents are put onto this network, each node is influenced by the fixed number $m$ of neighbors with it had selected when joining the network. It is not influenced by other nodes that selected it as neighbor after it joined the network, i.e., the neighbor relations were such that if A has B as a neighbor, $\mathrm{B}$ in general does not have $\mathrm{A}$ as a neighbor in the later interactions of agents on this DBA network.

\subsection{Directed and undirected Erdös-Rényi (DER, UER) random graphs}

An Erdös-Rényi (ER) random graph is formed by a set of $N$ vertices (sites) connected by $K$ links (bonds). ${ }^{17}$ With a probability $p$, a given pair of sites is connected by a bond $p=2 K / N(N-1)$. The connectivity of a site is defined as the total number of bonds connected to it, like $k_{i}=\sum_{j} l_{i j}$, where $l_{i j}=1$ if there is a link between the sites $i$ and $j$ and $l_{i j}=0$ otherwise. Random graphs are completely characterized by the mean number of bonds per site, or the average connectivity $z=p(N-1)$. These links can be directed or undirected as well.

\subsection{Directed and undirected Appolonian $(D A N, U A N)$ networks}

The Appolonian network (AN) is composed of $N=3+\left(3^{n}-1\right) / 2$ nodes, where $n$ is the generation number and $N$ the node number. ${ }^{18,19}$ On these AN structures, we can introduce a disorder in such a way that we redirect a fraction $p$ of the links. This redirecting results in a directed network, preserving the outgoing node of the redirected link but changing the incoming node. When $p=0$, we have the standard AN networks, while for $p=1$, we have something similar to random networks. ${ }^{10} \mathrm{In}$ this procedure of the redirecting links, the number of outgoing links of each node is preserved even when $p=1$ and the network still have hubs that are the most influent nodes. These networks display a scale-free degree distribution and a hierarchical structure. In the undirected case, there exists the reciprocity of redirected link.

\subsection{Small-world networks}

Small-world (SW) networks are intermediate between the regular local networks and the random networks. They have two interesting features: one is high clustering which is the characteristic of regular networks, and the other is short path length that is characteristic of random networks. The combination of these two features suggests that SW networks can be used to describe the behavior of various real 
systems that present interactions between nodes, agents or people as social and economics systems. Here, we briefly describe the directed SW (DSW) ${ }^{11}$ and undirected SW (USW) networks ${ }^{10}$ used in this study as previously mentioned.

\section{- $D S W$ networks}

In the SW networks, we can introduce an asymmetric disorder in such a way that we redirect a fraction $p$ of the links. This redirecting results in a directed network, preserving the outgoing node of the redirected link but changing the incoming node, i.e., when $\mathrm{A}$ is tied to $\mathrm{B}, \mathrm{B}$ may not be linked to A but to someone else instead. When $p=0$, we have the SL in two dimensions, while for $p=1$, we have something similar to random networks. ${ }^{21}$

- USW networks

Different from directed case, there exists a reciprocity or symmetry of redirected links, i.e., if node A selects node B as incoming neighbor, then A is also an incoming neighbor, of $\mathrm{B}$. The neighbor relations were such that if $\mathrm{A}$ has $\mathrm{B}$ as a neighbor, $\mathrm{B}$ has $\mathrm{A}$ as a neighbor.

\subsection{Stauffer-Hohnisch-Pittnauer (SHP) networks}

Hohnisch bonds of SHP networks ${ }^{23-25}$ are links connecting nodes with different values (spins, opinions, etc.) on them; they are at each time step with a low probability 0.0001 replaced by a link to another randomly selected node. Links connecting agreeing nodes are not replaced. In the present work, we start with each node having links to four randomly selected neighbors. Thus, our SHP networks are similar to Small-World (Watts-Strogatz) networks but start from a random network instead of a square lattice and use opinion-dependent (instead of random) rewiring. All links are directed. ${ }^{23,24}$

\section{Zaklan Model and Evolution Dynamics}

The Zaklan model ${ }^{7}$ consists of agents located on nodes or sites of a regular or irregular structure. Each agent is represented by an individual spin $\sigma_{i}= \pm 1$, who can either be an honest taxpayer +1 or a cheater -1 . Initially, everybody is assumed honest. In each iteration, individuals can reconsider their behavior to become the opposite type of agent they were in the previous period. Each agent's environment may prefer tax evasion or reject it. The agent depends on two factors: First, the agent's environment exerts influence on the agent in the next period. Second, people's decisions are partly autonomous, independent of their environment. This autonomous part is responsible for the emergence of tax evasion because some initially honest taxpayers decide to evade taxes and then exert influence on others to do so as well. Taxpayers have the greatest influence to turn honest citizens into tax cheaters if cheaters constitute a majority in the respective neighborhood. On the other hand, if most people in the vicinity are honest, the respective individual is likely to become a taxpayer if (s)he was a tax cheater before. The model also includes an enforcement 
mechanism that consists of two components: a probability of an efficient audit $p_{a}$ and a punishment time $k$. If tax evasion is detected, the individual must remain honest for a number $k$ of periods to be specified. One iteration is one sweep through the entire lattice. The temporal evolution of this model can be performed by using an equilibrium or by nonequilibrium dynamics.

\subsection{Zaklan model via nonequilibrium dynamics of MVM}

The temporal evolution of the Zaklan model via MVM on various toplogies is performed as follows: initially, everybody is assumed honest (1) and in each time period, the system evolves by a single spin-flip dynamics and (s)he can keep himself honest taxpayer +1 or a cheater -1 with a switching probability $w$ given by

$$
w\left(\sigma_{i}\right)=\frac{1}{2}\left[1-(1-2 q) \sigma_{i} S\left(\sum_{\delta=1}^{k_{i}} \sigma_{i, \delta}\right)\right],
$$

where $S(x)$ is the sign \pm 1 of $x$ if $x \neq 0, S(x)=0$ if $x=0$, and the summation runs over all $k_{i}$ nearest-neighbor sites $\sigma_{i, \delta}$ of $\sigma_{i}$. Here, an agent assumes the value \pm 1 depending on the opinion of the majority of its neighbors. The noise parameter $q$ plays the role of the "Social temperature" in equilibrium systems and measures the probability of aligning $\sigma_{i}$ antiparallel to the majority of its neighbors $\sigma_{i, \delta}$. "Social temperature" generally measures the amount of randomness or noise in the system. If the quantity $E$ measures the deviation of the systems's behavior from a noiseless behavior, and if the probability distribution $P(\mathrm{E})$ for this "energy" is proportional to a Boltzmann-type exponential function $\exp (-E / T)$, then this is the definition of the "social temperature". In the present Zaklan-type models, the noiseless behavior means that taxpayers follow the majority of their neighbors in cheating or being honest, and the "social temperature" $T$ allows for noise, i.e., for deviations from the majority rule and thus for a stationary equilibrium of honesty and cheating. For the case of four neighbors and $q=0$, if three or four neighbors disagree with the central site, the center flips; if one or none disagrees, the center does not flip; if two agree and two disagree, it flips with probability $1 / 2$. If $q>0$, the center may disobey this majority rule and follow its own opinion or the orientation of the external rumors that act on a people community.

\subsection{Zaklan model via nonequilibrium dynamics of SRL model}

In the temporal evolution of the Zaklan model via SRL model on DSW network, the system dynamics traditionally is as follows. We assign a spin variable $\sigma$ with values \pm 1 to each node of the network. At each step, we try to spin flip a node. The flip is accepted with some probability explained below. We put spin variables $\sigma_{i}$ taking the values \pm 1 and situated on every site $i$ of a DSW network with $N=L \times L$ sites, where $L$ is the side of the SL. In this model proposed by Sánchez et al., ${ }^{11}$ we start from a SL consisting of sites linked to their $l=4$ nearest neighbors by both outgoing 
and incoming links. Then, with rewiring probability $p$, we replace the nearest neighbor outgoing links by new outgoing links to different sites chosen at random, leaving the incoming links unchanged. After repeating this process for every link, we are left with a network with a density $p$ of SW directed links. Therefore, with this procedure, every site will have $l=4$ outgoing links and a varying (random) number of incoming links as shown in Fig. 1. Then, the spins (people, economic agents) are placed at the network sites. Thus, any agent is connected by $l$ outgoing links to other agents or mates and can be in one of two possible $\sigma_{i}$ states taking the values \pm 1 . Depending on the state of its mates (outgoing links) in the neighborhood, an agent may change its state according to a majority rule (ferromagnetic). To implement this, we introduce the payoff function $E_{i}$ :

$$
E_{i}=2 \sigma_{i} \sum_{j=1}^{l} \sigma_{j},
$$

where the sum is carried out over the $l$ mates of agent $i$. The external noise or social temperature $T$ is included allowing some degree of randomness in the time evolution. Then, for a given value of the external social temperature, the update of the model is performed as follows: At each step, an agent (network site) is randomly chosen and its corresponding $f_{i}$ is computed according to Eq. (1).

(i) If $E_{i}<0, \sigma_{i} \rightarrow-\sigma_{i}$. The agent $i$ opposes the neighborhood majority of its mates and the change of its actual state is accepted,

(ii) If $E_{i}>0$, the change of its actual state is accepted with the probability

$$
P_{i}=e^{-E_{i} / T},
$$
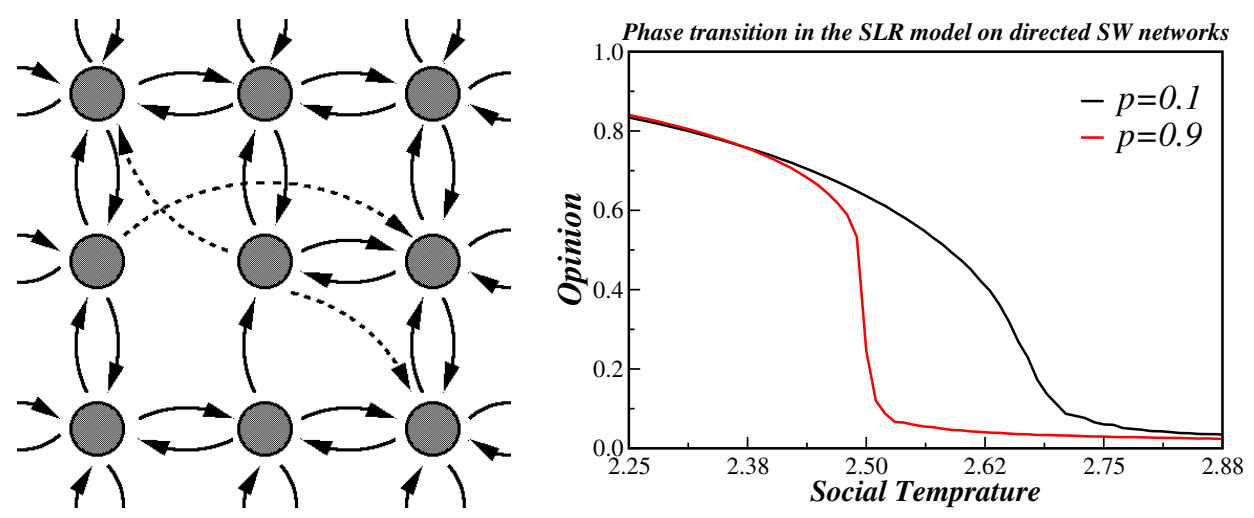

Fig. 1. On the left, we show a DSW network in two dimensions where the dotted lines represent rewired links. ${ }^{11}$ On the right, we show two types of phase transition. For rewiring probabilities $p=0.1$ (black line), the SLR model presents a continuous phase transition and a discontinuous phase transition for $p=0.9$ (red line). 
which depends on temperature. Therefore, this model is a nonequilibrium model, since detailed balance is not satisfied, due to the directedness of the links. At $p=p_{c}$ : tricritical point, occurs a change in the phase transition form. This changes from second-order to first-order transition with increasing $p$. The simulations have been performed on DSW networks sizes of $N=400$ of sites (nodes or agents). The opinion of a people community per total number of people is given by

$$
O(T)=\frac{\sum_{i} \sigma_{i}}{N}
$$

In order to model tax evasion, we further use for all agents one probability $p_{a}$ of an efficient audit. If tax evasion, $\sigma_{i}=-1$, is detected by this audit, the agent must remain honest, $\sigma_{i}=1$, for a number $k$ of time steps. Again, one time-step is one sweep through the entire network. In Fig. 1 (right), we show two types of phase transitions due Eq. (3). For rewiring probabilities $p=0.1$ (black line), the SRL model presents a continuous phase transition, while for $p=0.9$ (red line), we see a discontinuous phase transition. In the case $p=0.1$, the opinion of the agents or people about some subject change smoothly, with growing temperature close to a critical point, from an organized state to another disorganized one with the growth of social temperature $(T)$. Here, organized state means that most agents are of the same opinion (honest or dishonest) and disorganized state means that half of the agents are honest and the other half are dishonest. Instead, for $p=0.9$, the phase transition occurs abruptly, i.e., a jump from a organized to disorganized state near the critical temperature $\left(T_{c}\right)$ of $p=0.9$, i.e., the opinion of a people community jumps to the opposite opinion, next to $T_{c}$.

\section{Controlling the Tax Evasion Dynamics}

The fraction of tax evaders is

$$
\operatorname{tax} \text { evasion }=\frac{\left[N-N_{\text {honest }}\right]}{N},
$$

where $N$ is the total number and $N_{\text {honest }}$ the honest number of agents. The tax evasion is calculated at every time to step $t$ of system evolution.

Here, we first will present the baseline case $k=0$ and $p_{a}=0 \%$, i.e., no use of enforcement, for different network structure. We use $q=0.95 q_{c}$ for DBA, UBA and SHP networks (where $q_{c}=0.431, q_{c}=0.306$, and $q_{c}=0.166$, respectively), AN $\left(q_{c} \simeq 0.18\right)$, DER and UER $\left(q=0.9 q_{c}=0.175\right.$ and $\left.q_{c}=0.181\right)$ and for SRL on DSW, we set $T=0.95 T_{c}$ with $T_{c}=2.7(p=0.1)$ and $T_{c}=2.5(p=0.9)$. All simulations are performed over 20,000 time steps. For very low noises, the part of autonomous decisions almost completely disappears. The individuals then base their decision solely on what most of their neighbors do. A rising noise has the opposite effect. Individuals then decide more autonomously. 


\section{Results}

In Fig. 2, we plot tax evasion for DBA and UBA with $N=400$ agents for different enforcement $k$ and audit probability $p_{a}$. For case (a) and (d), we plot the baseline case $k=0$ and $p_{a}=0$, i.e., no use of enforcement for DBA and UBA. In cases (b) and (e), we show the tax evasion level decreases, on DBA and UBA, for a more realistic set of possible values degrees of punishment $k=10$ and audit probability $p_{a}=4.5 \% \cdot{ }^{36,7}$ In cases (c) and (f), we also show the tax evasion level decreases much more for an extreme set of punishment $k=50$ and audit probability $p_{a}=90 \%{ }^{7}$ All simulations were performed over 20,000 time steps. Here, we can see that the tax evasion level decreases more on UBA than DBA. This is due to the high degree of connectivity of UBA in relation to DBA.

In Fig. 3, we also plot tax evasion, but now for DER and UER with $N=400$ agents and the same parameter set used in the Fig. 2. In this case, the results are the same for both DER and UER, i.e., the high degree of connectivity of UER in relation to DER is irrelevant.

In Fig. 4, we plot tax evasion on $\mathrm{ANs}$ and SHP, with the same parameter set used in the Fig. 2. In this case, the results for tax evasion level decreases more on SHP than ANs.

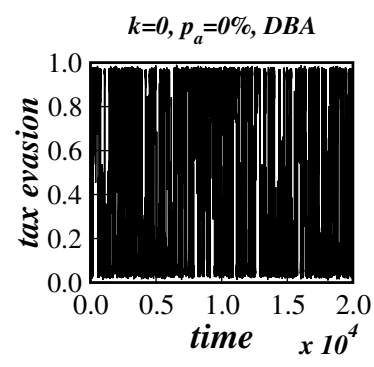

(a)

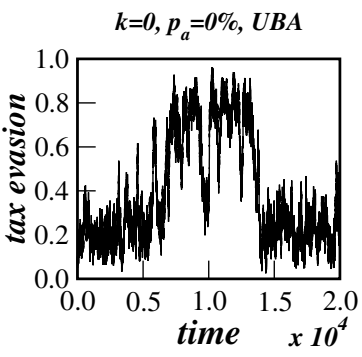

(d)

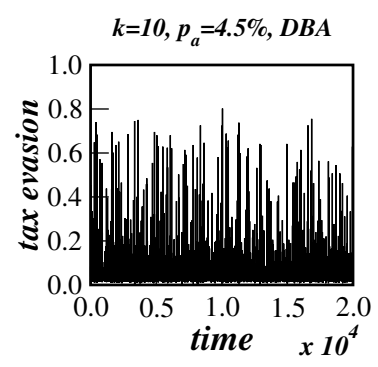

(b)

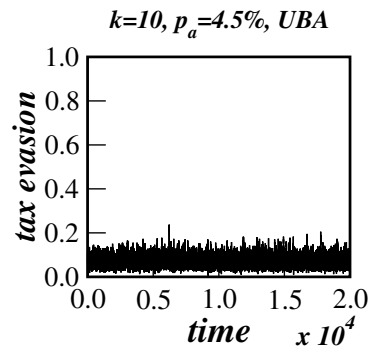

(e)

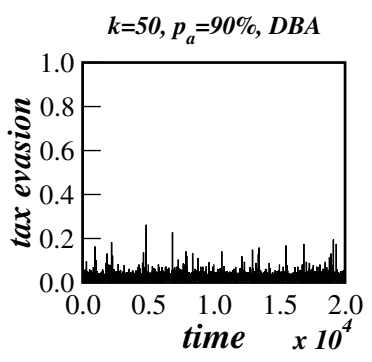

(c)

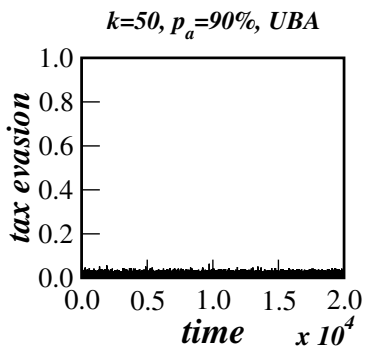

(f)

Fig. 2. Baseline case: $p_{a}=0 \%$ and $k=0$ in (a) and (d). In (b) and (e) we have the realistic case with punishment $k=10$ and audit probability $p_{a}=4.5 \%,{ }^{36,7}$ and for (c) and (f) we have an extreme case of punishment $k=50$ and audit probability $p_{a}=90 \% .^{7}$ We perform all simulations over 20,000 time steps, also in the later figures. 


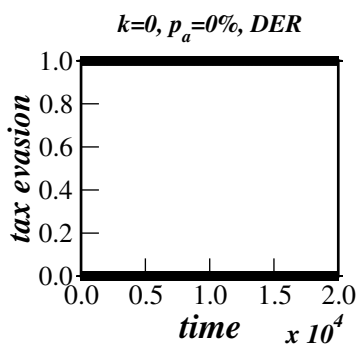

(a)

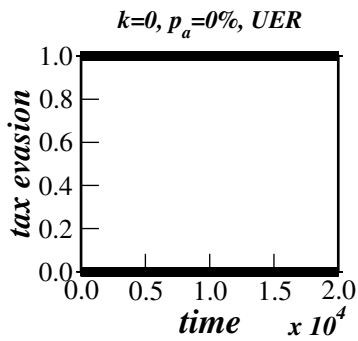

(d)

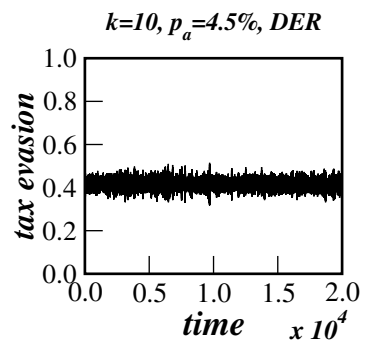

(b)

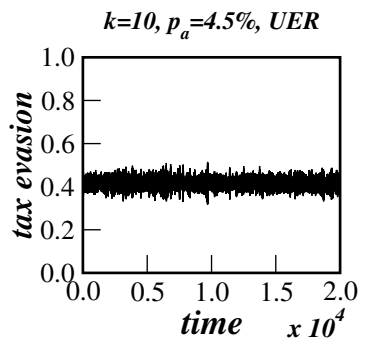

(e)

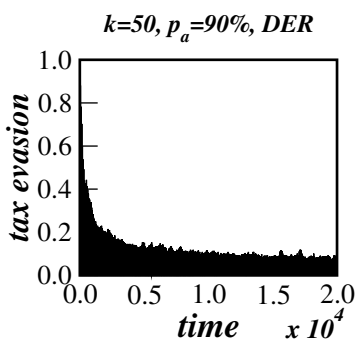

(c)

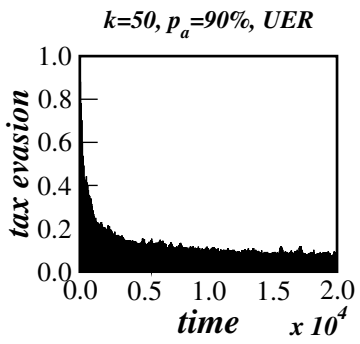

(f)

Fig. 3. The same simulation design as in Fig. 2, but now on DER and UER.

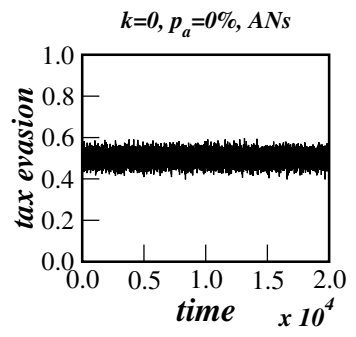

(a)

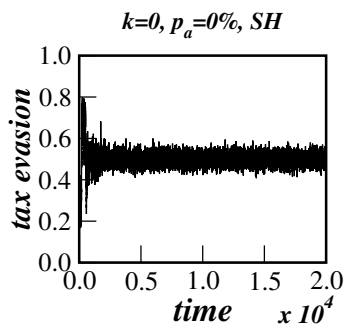

(d)

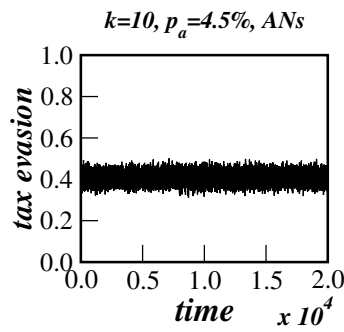

(b)

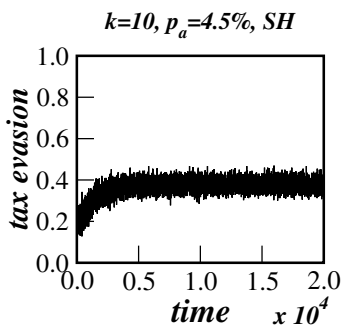

(e)

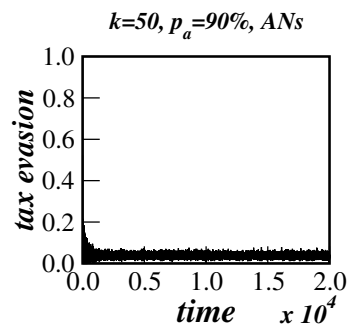

(c)

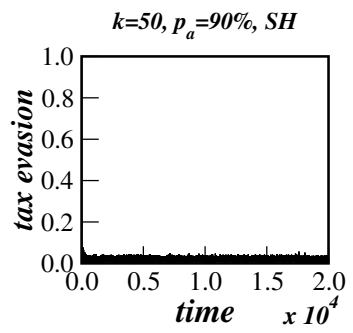

(f)

Fig. 4. The same simulation design as in Fig. 2, but now on ANs and SHP. 


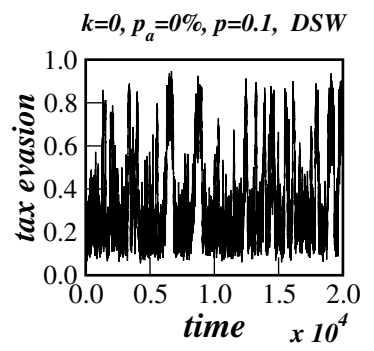

(a)

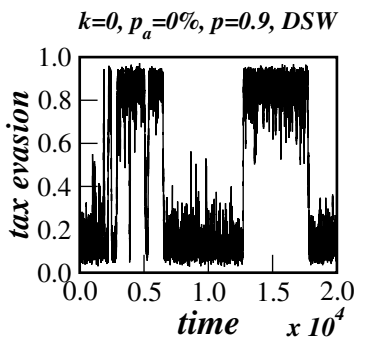

(d)

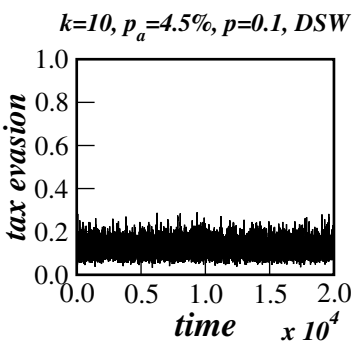

(b)

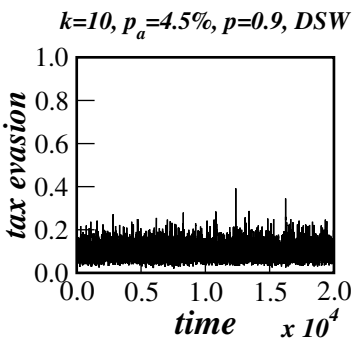

(e)

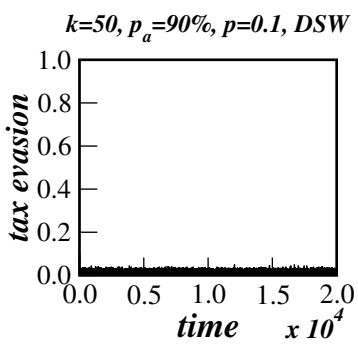

(c)

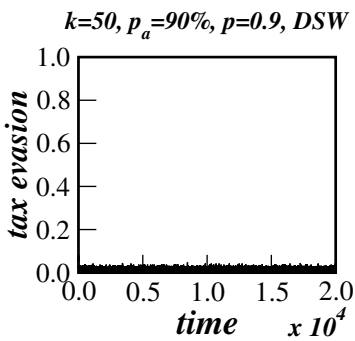

(f)

Fig. 5. The same simulation design as in Fig. 2, but now on DSW for $p=0.1$ and 0.9 .

In Fig. 5, we plot tax evasion on DSW for both rewiring probability $p=0.1$ and 0.9 , with the same parameter set used in the Fig. 2. In this case, the results for tax evasion level are independent of whether $p=0.1$ or 0.9 . For the SRL model on DSW, it is known that for $p>p_{c} \simeq 0.65$, the phase transition is of first-order, i.e., a jump occurs where most honest people flip to cheating for $p=0.9$; while for $p<p_{c}$, the phase transition is of second-order, i.e., the honest people flip to cheating smoothly, for $p=0.1$. Because of this behavior, we set a fixed noise $(T)$ to some values slightly below $T_{c}$, where the case that agents distribute in equal proportions onto the two alternatives is excluded. We set $T=0.95 T_{c}$ with $T_{c}=2.7(p=0.1)$ and $T_{c}=2.5$ $(p=0.9)$ such that we see flips of the whole system in the baseline cases $k=p_{a}=0$. Then we vary the degrees of punishment (10 and 50) and audit probability rate $\left(p_{a}=4.5 \%\right.$ and 90\%). If tax evasion is detected by an audit, the enforcement mechanism $p_{a}$ and the time of punishment $k$ control the tax evasion level.

\section{Conclusion}

Following Wintrobe and Gërxhani, ${ }^{36}$ less developed countries may have more tax evasion because of less trust in government. To understand this problem, Zaklan et $a .^{7,8}$ proposed a model, called Zaklan model, using Monte-Carlo simulations and an equilibrium dynamics (Ising model) on square lattices. Their results are in good 
agreement with analytical and experimental results obtained by. ${ }^{36}$ In this work, we show that the Zaklan model of tax evasiom is very robust because we use a nonequilibrium dynamics (MVM and SRL) to simulate the Zaklan model, with results similar to equilibrium dynamics (Ising model), ${ }^{7,8}$ and also on various topologies. ${ }^{35}$ Also here we found the plausible result that tax evasion is diminished by higher audit probability $p_{a}$ and stronger punishment $k$.

\section{Acknowledgments}

The author thanks Dietrich Stauffer for many suggestions and fruitful discussions in the development this work. He also acknowledges the CNPQ for its financial support. This work also was supported the system SGI Altix 1350 the computational park CENAPAD.UNICAMP-USP, SP-BRAZIL and Dietrich Stauffer Computational Physics Lab-TERESINA-PIAUÍ-BRAZIL.

\section{References}

1. K. Bloomquist, Soc. Sci. Comput. Rev. 24 (2006) 411.

2. J. Andreoni, B. Erard and J. Feinstein, J. Econ. Lit. 36 (1998) 818.

3. L. Lederman, Public Law Research paper No. 50, (2003).

4. J. Slemrod, J. Econ. Perspective 21 (2007) 25.

5. S. Gächter, Discussion Papers 2006-03, CeDEx, University of Nottingham (2006).

6. B. S. Frey and B. Togler, Tax morale and conditional cooperation, J. Comp. Econ. 35(1) (2007) 136.

7. G. Zaklan, F. Westerhoff and D. Stauffer, J. Econ. Interact. Coor. 4 (2009) 1.

8. G. Zaklan, F. W. S. Lima and F. Westerhoff, Physica A 387 (2008) 5857.

9. M. J. Oliveira, J. Stat. Phys. 66 (1992) 273.

10. M. E. J. Newman, S. H. Strogatz and D. J. Watts, Phys. Rev. E 64 (2001) 026118.

11. A. D. Sanchez, J. M. Lopez and M. A. Rodriguez, Phys. Rev. Lett. 88 (2002) 048701.

12. A.-L. Barabási and R. Albert, Science 286 (1999) 509.

13. A. Aleksiejuk, J. A. Hołyst and D. Stauffer, Physica A 310 (2002) 269.

14. M. A. Sumour and M. M. Shabat, Int. J. Mod. Phys. C 16 (2005) 585.

15. M. A. Sumour, M. M. Shabat and D. Stauffer, Islam. Univ. J. (Gaza) 14 (2006) 209.

16. F. W. S. Lima and D. Stauffer, Physica A 359 (2006) 423.

17. P. Erdös and A. Rényi, Publ. Math. Debrecen 6 (1959) 290; P. Erdös, A. Rényi, Publ. Math. Inst. Hung. Acad. Sci. 5 (1960) 17; P. Erdös and A. Rényi, Bull. Inst. Int. Stat. 38 (1961) 343.

18. J. S. Andrade Jr., H. J. Herrmann, R. F. S. Andrade and L. R. da Silva, Phys. Rev. Lett. 94 (2005) 018702.

19. R. F. S. Andrade and H. J. Herrmann, Phys. Rev. E 71 (2005) 05613.

20. B. Balobás, Random Graphs (Academic Press, New York, 1985).

21. D. J. Watts and S. H. Strogatz, Nature 393 (1998) 440.

22. S. Wasseman, K. Faust and B. Balobás, Social Networks Analysis (Cambridge University Press, Cambridge, 1994).

23. F. W. S. Lima, T. Hadzibeganovic and D. Stauffer, Physica A 388 (2009) 4999.

24. T. Qiu, T. Hadzibeganovic, G. Chen, L.-X. Zhong and X.-R. Wu, Comput. Phys. Comm. 181 (2010) 2057.

25. D. Stauffer, M. Hohnisch and S. Pittnauer, Physica A 370 (2006) 734. 
26. F. W. S. Lima, Int. J. Mod. Phys. C. 17 (2006) 1267.

27. F. W. S. Lima, Commun. Comput. Phys. 2 (2007) 358.

28. F. W. S. Lima, Int. J. Mod. Phys. C 19 (2008) 1063.

29. F. P. Fernandes and F. W. S. Lima, Int. J. Mod. Phys. C 19 (2008) 1777.

30. F. W. S. Lima and G. Zaklan, Int. J. Mod Phys. C 19 (2008) 1797.

31. F. W. S. Lima, T. Hadzibeganovic and D. Stauffer, Physica A 388 (2009) 4999.

32. F. W. S. Lima, J. Phys. Conf. Ser. 246 (2010) 012033.

33. S. Hokamp and M. Pickardt, Int. Econ. J. 24 (2010) 541.

34. M. Pickhardt and G. Seibold, Income tax evasion dynamics: Evidence from an agent-based econophysics model, J. Econ. Psychol. 40 (2014) 147.

35. F. W. S. Lima, Analysing and controlling the tax evasion dynamics via majority-vote model, J. Phys. Conf. Ser. 246 (2010) 012033.

36. R. Wintrobe and K. Gërxhani, Tax Evasion and Trust: A Comparative Analysis, in Proc. Annual Meeting of the European Public Choice Society (Berlin, 2004), p. 15. 